\title{
Housing improvements for health and associated socio-economic outcomes: evidence from a Cochrane Review and its application to Sri Lanka
}

\author{
Sumudu Avanthi Hewage ${ }^{1^{*}}$ \& Chrishantha Abeysena ${ }^{2}$ \\ ${ }^{1}$ Postgraduate Institute of Medicine, University of Colombo, Sri Lanka; ${ }^{2}$ Department of Public Health, Faculty of \\ Medicine, University of Kelaniya, Sri Lanka \\ *Correspondence: sumuduavanthi@gmail.com
}

DOI: https://doi.org/10.4038/jccpsl.v23i4.8114

Received on: 7 August 2017

Accepted on: 5 December 2017

\section{Introduction}

An updated Cochrane Review concluded that certain aspects of mental and physical health of inhabitants benefit from housing improvements. Targeted interventions aimed at those with poor health show greater health improvements, rather than programmes that deliver improvements across areas and do not target according to the levels of individual needs. Near absence of local data on health related outcomes secondary to poor housing conditions limits the assessment of the impact of these interventions in Sri Lanka. However, the World Health Organization estimation of 4300 deaths attributable to indoor air pollution in Sri Lanka in 2004 warrants action to improve housing conditions for the betterment of community health. This article discusses the findings of a Cochrane Review on health and associated socioeconomic outcomes secondary to housing improvements and its implications for Sri Lanka.

Key words: housing improvements, physical and mental quality of life, social wellbeing, indoor air pollution, school performance

\section{Relevance}

The link between poor housing and poor health has been repeatedly proven in the literature across the globe. Poor air quality (particles and fibres), warmth and humidity, radon, noise, house dust mites, environmental tobacco smoke and fire have been identified as most commonly found significant housing hazards. Both physical and mental health has shown to be affected by these hazards. These include respiratory symptoms, bronchial asthma, injury and death from accidents and fire, depression and anxiety, hypothermia, skin and eye irritation, and general health symptoms (1). The World Health Organization (WHO) estimated that the number of deaths attributable to indoor air pollution in Sri Lanka was 4300 in 2004 (2). As such, this evidence is important for public investment which could reduce mortality and lead to better health in the community in Sri Lanka. In doing so, it is important to assess which interventions in housing improvements are proven to benefit the health of inhabitants.

\section{Current best evidence}

The Cochrane Review by Thomson et al (2013) summarizes the evidence available on housing improvements for health and associated socioeconomic outcomes up to date (1). Objectives of the review were to assess the health and social impacts on residents following improvements in housing conditions. Experimental as well as uncontrolled studies which have assessed any health outcome following housing improvement have been included. The review analysed 39 such studies from any region of the world including both industrialized and non-industrialized countries, of which the summary is given in Table 1 . 
Table 1. Summary of the participants, interventions and outcomes assessed in primary studies evaluated for the Cochrane Review

\section{Types of participants}

House types

Housing programmes
Permanent residences for independent living; sheltered housing; residential care homes

Discrete housing programmes i.e. where a pre-defined housing improvement is delivered to a pre-defined population, either at area level or assessed according to individual eligibility
Housing interventions
Rehousing where it clearly involves improvements in indoor housing conditions due to improved structure or housing fabric. This may include emergency rehousing e.g. following natural disaster, and medical priority rehousing where there is physical improvement in housing conditions delivered beyond adaptations to meet mobility, care or mental health needs, structural improvements, and warmth and energy efficiency improvements

\section{Types of interventions}

Housing interventions were defined as re-housing and any physical change to housing infrastructure
- Warmth and energy efficiency improvements (post-1985)

- Rehousing or retrofitting \pm neighbourhood renewal (post-1995)

- Provision of basic housing in low or middle income countries (post-1990)

- Rehousing from slums (pre-1970)

\section{Outcomes}

Primary outcome

Any measure which could be interpreted as a direct measure of health or mental and physical illness, self-reported well-being and quality of life.

Secondary outcomes

Social and socio-economic outcomes which could be interpreted as determinants of health, such as household income, measures of social contact, social exclusion, education, employment and time off work have been considered as secondary outcomes.

Outcomes for both adults and children were eligible for inclusion in the review and there was no minimum follow-up period to assess health effects. The final impact in studies where outcomes were assessed at multiple time points was considered as the study's findings.

\section{Risk of bias assessment}

Risk of bias (RoB) assessment has been carried out to assess the quality of the primary studies included in the review across the domains of random sequence generation, allocation concealment, blinding of participants and personnel, blinding of outcome 
assessment, blinding of analysts, incomplete outcome data, selective reporting, baseline outcome characteristics similarity, baseline characteristic similarity, contamination, baseline response and implementation of intervention. Quality of the included studies was poor according to the RoB assessment. None of the studies was rated as having 'low' RoB across all domains. Poor reporting resulted in many studies classifying as unclear RoB. Overall assessment of quality demonstrated variation across the studies.

\section{Results}

A total of 39 studies which reported quantitative or qualitative data or both were included in the review. Extreme heterogeneity in methods as well as in the interventions, samples, context and outcome prevented meta-analysis of data leaving very little quantitative synthesis. Data were therefore synthesized narratively. Interventions were grouped into broad categories as mentioned below, based on the type of housing intervention, context and study population.

- Rehousing from slums (pre-1970)

- Warmth and energy efficiency improvements (post-1985)

- Provision of basic housing in low and middle income countries (post-1990)

- Rehousing and retrofitting with or without neighbourhood renewal (post-1995)

Older studies (pre-1970) examined the changes in health following rehousing from slums, including relocating poor families from slum conditions to improved or new housing in a new neighbourhood. One overall good quality study reported improvement in six measures of mental health one year after rehousing. Mental health improvements were seen in negative mood, dissatisfaction with status quo, potency, pessimism, emotionality and nervousness. General health impacts or respiratory health impacts were not reported in those studies.

Data from studies of warmth and energy efficiency interventions (post-1985) suggested that improvements in general health, respiratory health and mental health are possible. Warmth improvements were associated with increased usable space, increased privacy and improved social relationships. Absenteeism from work or school due to illness was also reduced. Out of 15 studies, five were randomized controlled trials while seven were overall good quality studies.

Studies on the provision of basic housing in lowand middle-income countries (post-1990) included provision of sealed double pit-water latrines to households and communal water hand pumps to a village where provision was low, repairs to housing as well as wider neighbourhood improvements to water and sanitation infrastructure, street lighting and repair of public buildings. All were neither experimental nor good quality studies. Evidence from these studies is limited with respect to quantity and quality.

The housing improvements in high income countries (post-1995) included refurbishment, rehousing, relocation, installation of central heating and insulation. The interventions were similar being government investment to improve housing conditions in deprived areas, predominantly among social housing. All were observational studies. Some were good quality studies. Impacts following housing-led neighbourhood renewal were less clear; these interventions targeted areas rather than individual households in dire need. Area-based housing improvement programmes, for example, programmes of housing-led neighbourhood renewal, which improve housing regardless of the individual needs, may not lead to clear improvements in housing conditions for all the houses in a neighbourhood. This may explain why health improvements following these programmes are not always apparent.

\section{Aptness of evidence to Sri Lanka}

Interventions which are applicable to the local setting need to be chosen carefully after analysing the local housing conditions. For example, measures to improve temperature and humidity of living rooms will not be necessary in the local context, whereas measures to improve the indoor air quality will have a great yield. Limited literature on health related impacts due to poor housing conditions other than indoor air quality is a major drawback in determining the most effective interventions applicable to Sri Lanka. With the political commitment available for housing construction in Sri Lanka, implementing recommendations is feasible, and it can be safely assumed that the acceptability from the policy level as well as from the public will be positive. 


\section{Implications for policy planning}

Overall evidence suggests that improvements in housing conditions can lead to improvements in health. Health improvement is most likely when the interventions are targeted at those with poor health and inadequate housing conditions. In Sri Lanka, 1.5\% of all housing units are huts/shanties, $4.6 \%$ of households use river/tanks/streams as the source of drinking water and nearly $1.7 \%$ of houses do not use any type of sanitary facility (3). Nandasena et al (2011) found that indoor $\mathrm{PM}_{2.5}$ levels exceeded the recommended guidelines of WHO in all households studied in the Colombo Municipal Council area and in $70 \%$ of households using biomass in Panadura Medical Officer of Health area (4). Thus, interventions to improve housing conditions should be directed at locally relevant target communities.

\section{Implications for research}

High quality randomized controlled trials are needed from developing countries to assess the health and health related impacts of housing improvements in the context of local settings. Special emphasis should be made on reporting the studies, which is essential in replicating studies and in assessing the quality of the study.

\section{Conflict of interest None}

\section{References}

1. Thomson H, Thomas S, Sellstrom E, Petticrew M. Housing improvements for health and associated socio-economic outcomes. Cochrane Database of Systematic Reviews 2013; (2): CD008657.

2. WHO. Country Profile of Environment Burden of Disease: Sri Lanka 2009. Geneva: World Health Organization, 2009.

3. DCS. Sri Lanka Demographic and Health Survey 2012. Colombo: Department of Census and Statistics, 2012.

4. Nandasena S, Wickremasinghe AR, Sathiakumar N. Levels and determinants of indoor air quality in Sri Lankan households. Proceedings ISES 2011: Advancing Exposure Science for Environmental Health, International Society of Exposure Science, Baltimore, USA, 2011. 